\title{
Chemotherapy Resistance as a Predictor of Progression-Free Survival in Ovarian Cancer Patients Treated with Neoadjuvant Chemotherapy and Surgical Cytoreduction Followed by Intraperitoneal Chemotherapy: A Southwest Oncology Group Study
}

\author{
Amy D. Tiersten ${ }^{a}$ James Moon ${ }^{b}$ Harriet O. Smith ${ }^{c}$ Sharon P. Wilczynski ${ }^{d}$ \\ William R. Robinson, 3rd ${ }^{\mathrm{e}}$ Maurie Markman $^{\mathrm{f}}$ David S. Alberts $^{\mathrm{g}}$ \\ ${ }^{a}$ New York University Cancer Center, New York, N.Y., b Southwest Oncology Group Statistical Center, Seattle, Wash., \\ 'University of New Mexico, Albuquerque, N. Mex., 'City of Hope National Medical Center, Duarte, Calif.,

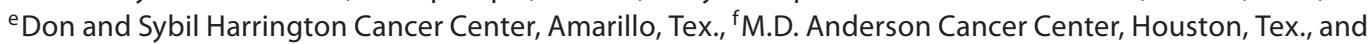 \\ gUniversity of Arizona Cancer Center, Tucson, Ariz., USA
}

\section{Key Words}

Paclitaxel - Carboplatin • Ovarian cancer - Cytoreduction •

Neoadjuvant chemotherapy

\begin{abstract}
Purpose: In vitro testing of the activity of chemotherapeutic agents has been suggested as 1 method to optimally select drugs for patients with ovarian cancer. There are limited prospectively obtained data examining the clinical utility of this approach. We sought to obtain a preliminary assessment of this strategy in a trial that examined the administration of neoadjuvant chemotherapy followed by surgical cytoreduction and intraperitoneal chemotherapy in women with advanced ovarian cancer. Methods: Women with stage III/IV epithelial ovarian carcinoma that presented with large-volume disease were treated with neoadjuvant intravenous paclitaxel and carboplatin for three 21-day cycles followed by cytoreductive surgery. If optimally debulked, patients re-
\end{abstract}

ceived intravenous paclitaxel, intraperitoneal carboplatin and intraperitoneal paclitaxel for six 28-day cycles. Tumor cloning assay results (Oncotech) were correlated with progression-free survival. Results: Sixty-two patients (58 eligible) were registered from March 2001 to February 2006. Thirty-six eligible patients had interval debulking and 26 received postcytoreduction chemotherapy. Twenty-two patients had tumor cloning assay results available. The clinical features of this population were similar to those of the larger group of women who entered this study. There was no difference in progression-free survival between patients whose cancers were defined as 'resistant' or 'nonresistant' to either platinum or paclitaxel. Conclusions: While the small patient numbers in this trial do not permit definitive conclusions, these data fail to provide support for the argument that prospectively obtained in vitro data regarding platinum or paclitaxel resistance will be highly predictive of clinical outcome in advanced ovarian cancer.

Copyright $\odot 2010$ S. Karger AG, Basel

\section{KARGER}

๑ 2010 S. Karger AG, Basel

Fax +41 613061234 E-Mail karger@karger.ch www.karger.com www.karger.com/ocl
Amy D. Tiersten, MD

New York University Cancer Center

160 E, 34 Street

New York, NY 10016 (USA)

Tel. +1 212731 5349, Fax +1 212731 5574, E-Mail amy.tiersten@nyumc.org 


\section{Introduction}

Standard therapy of advanced ovarian cancer involves initial surgical management to perform staging and maximal cytoreduction whenever possible. An alternative to conventional surgery followed by chemotherapy is to deliver neoadjuvant chemotherapy followed by surgery. Previous small studies of neoadjuvant chemotherapy have suggested similar progression-free survival (PFS) and overall survival, as compared with initial surgery followed by chemotherapy, with reduced surgical morbidity, better optimal debulking rates, less cost and better chemotherapy tolerability [1-6].

Intraperitoneal chemotherapy has been shown to improve survival in optimally debulked ovarian cancer patients [7-9]. In patients for whom optimal debulking is felt to be unlikely, 1 strategy could be to incorporate intraperitoneal therapy postoperatively in patients that are able to be optimally cytoreduced following neoadjuvant chemotherapy. The primary purpose of this study was to evaluate overall survival, PFS and toxicity in patients treated with this strategy as well as to evaluate the percent of patients that are able to be successfully cytoreduced to optimal disease following neoadjuvant therapy. The primary publication that includes additional details regarding study design and results was published in Gynecologic Oncology, March 2009 [10].

A secondary endpoint of this trial was to explore the relationship between in vitro resistance to carboplatin and paclitaxel and PFS. Previous published reports had suggested the extreme drug resistance (EDR) in vitro assay to be highly predictive of clinical resistance to chemotherapy [11]. One study correlated EDR assay results to survival in 40 ovarian cancer patients [12]. A significantly worse survival (28 months) for highly cisplatin-resistant patients was found as compared to low-resistant patients (51 months). Holloway et al. [13] have shown that a correlation of the EDR in vitro assay and patient outcome exists. In this study, patients with tumors demonstrating in vitro EDR to platinum were at significantly increased risk for progression and death when treated with standard platinum-based regimens.

Unfortunately, the majority of reports correlating clinical outcome with in vitro resistance studies have been retrospective in nature. Thus, there is an important need for the conduct of prospectively designed and performed studies to further evaluate the relationship between in vitro-defined resistance to cisplatin and/or paclitaxel and subsequent PFS and overall survival in patients with ovarian cancer.

\section{Patients and Methods}

Patients were considered eligible for this study if the clinical picture was consistent with stage III epithelial ovarian cancer, primary peritoneal or fallopian tube cancer with a large pelvic mass, bulky abdominal disease and/or malignant pleural effusion. Patients must have had adenocarcinoma by biopsy or cytology (nonmucinous histology) compatible with an epithelial ovarian, fallopian tube or peritoneal primary cancer. Patients were deemed unlikely to be able to be optimally cytoreduced by the evaluating gynecologic oncologist.

Patients who had undergone an exploratory laparotomy prior to enrollment were eligible only if an aggressive tumor debulking procedure was not performed (e.g. BSO/TAH with omentectomy). Patients were informed of the investigational nature of the study and gave their informed written consent in accordance with institutional and federal guidelines.

Neoadjuvant chemotherapy was given for three 21-day cycles and consisted of paclitaxel $175 \mathrm{mg} / \mathrm{m}^{2}$ over $3 \mathrm{~h}$ followed by carboplatin (target AUC 6) i.v. over 30-60 min. If following neoadjuvant chemotherapy, a patient had a $\geq 50 \%$ decrease in CA-125 from baseline, an exploratory laparotomy was performed with the goal of optimal debulking or complete resection of any residual tumor seen. At the completion of cytoreductive surgery, patients must have had optimal disease defined as no residual lesions after resection or residual disease such that no single lesion measured $\geq 1 \mathrm{~cm}$ in the largest diameter. Patients whose disease was not optimally cytoreduced were removed from the protocol treatment.

Postoperative chemotherapy consisted of paclitaxel 175 $\mathrm{mg} / \mathrm{m}^{2}$ i.v. over $3 \mathrm{~h}$ on day 1 followed by carboplatin $(\mathrm{AUC}=5)$ i.p. on day 1 and paclitaxel $60 \mathrm{mg} / \mathrm{m}^{2}$ i.p. on day 8 for six 28 -day courses.

The primary goal of the study was to evaluate PFS and overall survival in stages III and IV, bulky ovarian, peritoneal and fallopian tube cancer patients treated with this strategy.

A secondary endpoint was to explore the relationship between in vitro resistance to carboplatin and paclitaxel with PFS. The in vitro cytotoxic activities of carboplatin and paclitaxel were tested using previously described techniques for epithelial cancers with solid tumor samples minced into 2 - to $3-\mathrm{mm}$ pieces and then further digested in $8 \mathrm{ml}$ of $0.15 \%$ type I collagenase, $0.015 \%$ DNase mixture (Sigma, St. Louis, Mo., USA). The digest was filtered through $8 \times$ sterile gauze and washed with McCoy's 5A medium as the resulting cell suspension was examined and, if necessary, a 25- or 30-mm mesh filtration was used to obtain single-cell suspension. Depending on the quantity of ovarian carcinoma cells, cellular suspensions were exposed in triplicate to the drug, positive controls and negative controls continuously at $37^{\circ} \mathrm{C}$ in a $5 \% \mathrm{CO}_{2}$ humidified chamber in agarose using RPMI 1640 medium (Gibco, Grand Island, N.Y., USA) supplemented with $10 \%$ FBS, $3 \mathrm{~nm}$ vitamin C (Irvine), $50 \mathrm{U} / \mathrm{ml}$ catalase (Sigma), 100 $\mathrm{U} / \mathrm{ml}$ penicillin (Irvine), $0.04 \mathrm{ng} / \mathrm{ml}$ hydrocortisone (Irvine), 100 $\mathrm{mg} / \mathrm{ml}$ streptomycin (Irvine), $2 \mathrm{mM} \mathrm{L}$-glutamine (Irvine), $1 \times$ nonessential amino acids (Irvine) and $5 \mathrm{mg} / \mathrm{ml}$ transferrin (Sig$\mathrm{ma}$ ) at a concentration of approximately $125,000 \mathrm{mg} / \mathrm{ml}$. Previous studies using this technique revealed drug concentrations ranging from $1.0-100 \mathrm{mg} / \mathrm{ml}$ during continuous exposure. Tritiated thymidine was added at $72 \mathrm{~h}$. Tumor cell growth, measured as a percentage of the control, was determined at 5 days. Significant 
Table 1. EDR assay results

\begin{tabular}{|c|c|c|c|c|c|c|}
\hline \multirow[t]{2}{*}{ Assay result } & \multirow[t]{2}{*}{ Total } & \multirow[t]{2}{*}{ Events } & \multicolumn{4}{|l|}{ PFS rates } \\
\hline & & & $\begin{array}{l}12 \text {-month } \\
\%\end{array}$ & $\begin{array}{l}18 \text {-month } \\
\%\end{array}$ & $\begin{array}{l}\text { 24-month } \\
\%\end{array}$ & $\begin{array}{l}\text { median } \\
\text { months }\end{array}$ \\
\hline \multicolumn{7}{|l|}{ Carboplatin } \\
\hline Nonresistant & 7 & 3 & 86 & 57 & 57 & Not reached \\
\hline Resistant & 15 & 8 & 93 & 77 & 58 & 30 \\
\hline \multicolumn{7}{|c|}{$\mathrm{HR}^{*}=0.85(\mathrm{p}=0.81) 95 \% \mathrm{CI}: 0.22-3.20$} \\
\hline \multicolumn{7}{|l|}{ Paclitaxel } \\
\hline Nonresistant & 9 & 5 & 89 & 63.5 & 51 & 39 \\
\hline Resistant & 10 & 6 & 90 & 67.5 & 54 & 30 \\
\hline \multicolumn{7}{|c|}{$\mathrm{HR}^{*}=1.07(\mathrm{p}=0.92) 95 \% \mathrm{CI}: 0.32-3.53$} \\
\hline
\end{tabular}

growth inhibition (sensitivity) was defined as reduction to less than $30 \%$ of the control. Intermediate growth inhibition was defined as reduction to $30-50 \%$ of the control. Growth $>50 \%$ of the control was regarded as resistant. The doses of standard drugs used in the in vitro assays (i.e., carboplatin paclitaxel $5 \mathrm{mg} / \mathrm{ml}$ or $5.9 \mathrm{~mm}$ ) were relative approximations of clinically achievable drug concentrations as defined by the mean plasma concentration time product (AUC) following administration of standard doses for single-agent chemotherapy of gynecologic cancers.

Fresh tissue submission for this assay was strongly encouraged, but not required, for all patients in the study. An additional informed consent for this was obtained.

Cox model analyses were performed for PFS with the EDR assay result as the only predictor.

\section{Results}

Sixty-two patients were registered between March 2001 and February 2006. Four patients were ineligible (1 had extensive surgery, 2 had distant metastases, 1 had CA125 < 70). The median age for the patients in the study was 62.6 (31.5-79.8). Forty-three patients (74\%) had ovarian cancer, $14(24 \%)$ had primary peritoneal cancer and in 1 patient (2\%), the primary site was not reported. Forty-three patients (74\%) had stage III disease and 15 (26\%) had stage IV. Thirty-six eligible patients (62\%) had interval debulking surgery and 26 eligible patients received postcytoreduction chemotherapy.

For the 22 patients of whom we have tumor sample data, the median age was 62 years (47-77); 17 patients (77.3\%) had ovarian cancer and $5(22.7 \%)$ had peritoneal primary cancer. Thirteen patients (59\%) had stage III disease and 9 (41\%) had stage IV. Sixteen out of 22 (73\%) patients received postcytoreduction intraperitoneal chemo- therapy as per protocol (6 were suboptimally debulked and went out of the study to receive IV chemotherapy). Twenty out of 22 patients had debulking surgery and had their tumor samples obtained from this procedure. The other 2 patients had tissue obtained from the initial biopsy. Other than this higher debulking rate, which had to be the case in order to have the tissue specimens, the other characteristics matched those of the total study sample very well. Sixteen out of 20 patients (80\%) had postdebulking chemotherapy, which also corresponded well with the 26 patients (72\%) who underwent debulking surgery and received postcytoreduction chemotherapy.

EDR assay results for carboplatin resistance were available for 22 patients. Seven patients were found to be nonresistant to carboplatin and 15 were resistant. There was no significant difference between PFS at 12, 18 and 24 months and the median PFS values between these 2 groups (table 1). EDR assay results for paclitaxel resistance were available for 19 patients. Nine patients were found to be nonresistant to paclitaxel and 10 were paclitaxel-resistant. Again, there was no significant difference in PFS outcome parameters between these 2 groups (table 1).

\section{Discussion}

In the last decade, there has been a revolution in oncology surrounding the promise of targeted therapeutics and tailoring treatment based on particular molecular phenotypes. The possibility of further improving treatment by tailoring individual cytotoxic therapeutic agents demonstrated to be biologically active in vitro would be a very appealing concept. 
Retrospective studies have supported the clinical relevance of EDR testing in ovarian cancer. Holloway et al. [13] reported that women who were treated with agents falling into the EDR category on the basis of in vitro testing had significantly inferior survival compared with women who received agents that showed low drug resistance in vitro. Another study of 119 paired synchronous samples of epithelial ovarian cancer found congruent results for EDR in $89 \%$ of cases [14]. Loizzi et al. [15] demonstrated a significant survival advantage for ovarian cancer patients who were treated with assay-assisted therapy as compared to empiric treatment. Despite these promising results, as of today we have no evidence from randomized trials that the selection of drugs to treat epithelial ovarian cancer using EDR assays is superior to empiric treatment. Therefore, there is a need for prospectively designed and performed studies to further evaluate the relationship of in vitro resistance to cisplatin and paclitaxel and subsequent progression-free and overall survival.

Although EDR assay results in this study were not predictive of outcome, it is interesting to note the higher than expected proportion of patients found to be resistant in our study. This outcome may relate to the small numbers of patients in our study, but could theoretically reflect the patient population selected for this study (i.e., patients referred for neoadjuvant chemotherapy who had largevolume disease and were deemed 'unlikely to be debulked'). The degree of resistance to carboplatin of $68 \%$ may help explain the relatively poor prognosis of these patients, and suggests that patients who are 'unlikely to be debulked' at initial diagnosis may be predetermined, biologically, to experience an inferior outcome. Because 20 of the 22 specimens analyzed were obtained at the time of debulking surgery (2 from initial biopsy), it is certainly possible that the 3 cycles of chemotherapy given prior to debulking surgery may have altered the samples in a way that could affect the assay. It is possible that EDR results obtained prior to initiating any chemotherapy may have produced a different result.

Our study failed to demonstrate any correlation between EDR assay results and PFS. Due to the relatively small number of patients, it is certainly not possible to draw definitive conclusions. However, several strengths of the current analysis should be noted. First, the data were blindly and prospectively obtained and independently evaluated. Second, long-term follow-up of the treated patient population is available. Finally, based on our limited data, the lower limits for the $95 \%$ CI of the hazard ratios were 0.22 and 0.32 for carboplatin and paclitaxel, respectively. These results suggest that a highly predictive assay result, i.e., with hazard ratios of a magnitude $<0.22$ or $<0.32$, would be unlikely. Despite this fact, because of the potential clinical relevance of the predictive utility of in vitro drug sensitivity/resistance testing in ovarian cancer management, further prospective studies with larger numbers of patients are clearly warranted.

\section{Acknowledgements}

This investigation was supported in part by the following PHS Cooperative Agreement grant numbers awarded by the National Cancer Institute, DHHS: CA32102, CA38926, CA12213, CA46368, CA105409, CA13612, CA46441, CA35262, CA45560, CA22433, CA67575, CA12644, CA35176, CA86780, CA46368, CA58861, CA58723, CA52654, CA35281 and CA35261. This study was also supported in part by Oncotech.

\section{References}

1 Schwartz PE, Rutherford TJ, Chambers JT, et al: Neoadjuvant chemotherapy for advanced ovarian cancer: long-term survival. Gynecol Oncol 1999;72:93-99.

$\checkmark 2$ Jacob JH, Gershenson DM, Morris M, et al: Neoadjuvant chemotherapy and interval debulking for advanced epithelial ovarian cancer. Gynecol Oncol 1991;42:146-150.

-3 Lim JTW, Green JA: Neoadjuvant carboplatin and ifosfamide chemotherapy for inoperable FIGO stage III and IV ovarian carcinoma. Clin Oncol (R Coll Radiol) 1993;5: 198-202.

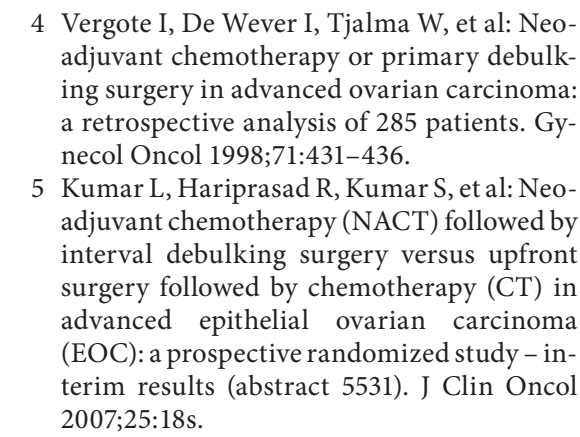

-6 Kuhn W, Rutke S, Späthe K, et al: Neoadjuvant chemotherapy followed by tumor debulking prolongs survival for patients with poor prognosis in International Federation of Gynecology and Obstetrics Stage IIIC ovarian carcinoma. Cancer 2001;92:25852591.

7 Alberts DS, Liu PY, Hannigan EV, et al: Intraperitoneal cisplatin plus intravenous cyclophosphamide versus intravenous cisplatin plus intravenous cyclophosphamide for stage III ovarian cancer. N Engl J Med 1996; 335:1950-1955. 
8 Markman M, Bundy B, Alberts D, et al: Phase III trial of standard-dose intravenous cisplatin plus paclitaxel versus moderately highdose carboplatin followed by intravenous paclitaxel and intraperitoneal cisplatin in small-volume stage III ovarian carcinoma: an intergroup study of the Gynecologic Oncology Group, Southwestern Oncology Group, and Eastern Cooperative Oncology Group. J Clin Oncol 2001;19:1001-1007.

>9 Armstrong DK, Bundy B, Wenzel L, et al: Intraperitoneal cisplatin and paclitaxel in ovarian cancer. N Engl J Med 2006;354:3443.
10 Tiersten AD, Liu PY, Smith HO, et al: Phase II evaluation of neoadjuvant chemotherapy and debulking followed by intraperitoneal chemotherapy in women with stage III and IV epithelial ovarian, fallopian tube or primary peritoneal cancer: Southwest Oncology Group Study S0009. Gynecol Oncol 2009; 112:444-449.

11 Fruehauf JP, Bosanquet AG: In vitro determination of drug response: a discussion of clinical applications. PPO Updates 1993;12: $1-10$.

12 Fruehauf JP, Saha S, Mehta RS, et al: Extreme drug resistance (EDR): assay to cisplatinum and survival in ovarian cancer patients - a comparative analysis. Program and $\mathrm{Ab}$ stracts Am Soc Clin Oncol 36th Annu Meet, New Orleans, 2000, abstract 1579.
13 Holloway RW, Mehta RS, Finkler NJ, et al: Association between in vitro platinum resistance in the EDR assay and clinical outcomes for ovarian cancer patients. Gynecol Oncol 2002;87:8-16.

14 Tewari K, Mehta RS, Burger RA, et al: Conservation of in vitro drug resistance profiles in epithelial ovarian cancer. Gynecol Oncol 2005;98:360-368.

15 Loizzi V, Chan JK, Osann K, et al: Survival outcomes in patients with recurrent ovarian cancer who were treated with chemoresistance assay-guided chemotherapy. Am J Obstet Gynecol 2003;189:1301-1307. 